Pacific Journal of Mathematics

PICONE-TYPE THEOREMS FOR HYPERBOLIC PARTIAL

KURT REIT 


\title{
PICONE-TYPE THEOREMS FOR HYPERBOLIC PARTIAL DIFFERENTIAL EQUATIONS
}

\author{
KURT KREITH
}

\begin{abstract}
Sturmian comparison theorems are established for hyperbolic partial differential equations of the form
\end{abstract}

$$
-\left(m(t) u_{t}\right)_{t}+\left(a(x) u_{x}\right)_{x}+p(x, t) u=0
$$

and

$$
-\left(M(t) v_{t}\right)_{t}+\left(A(x) v_{x}\right)_{x}+P(x, t) v=0
$$

when these equations are neither assumed to admit a separation of variables, nor to have equal principal parts. As such, the principal results constitute a generalization of the classical Sturm-Picone theorem.

1. Introduction. The fact that the Sturm-Picone theorem has a natural generalization to elliptic equations was observed by Picone [7] in 1911, was since rediscovered by several authors, and has recently been extended in several directions (see [11] for an extensive list of references), including consideration of equations of parabolic type. However, the question of an extension to hyperbolic equations has received relatively little attention in spite of the physical motivation (see §4) which exists.

One explanation for absence of an hyperbolic analogue is that the variational machinery implicit in the "Picone identity" does not carry over to hyperbolic equations. Rather, the indefiniteness of the quadratic form associated with a hyperbolic equation precludes the development of the disconjugacy criteria which are naturally associated with the Legendre sufficiency condition. For this reason generalizations to hyperbolic equations have been restricted to problems which allow for a separation of variables [2], [3], [10], [12] or to analogues of the more restrictive Sturm comparison theorem for equations with the same principal parts [4].

In this paper we seek to develop a more satisfactory hyperbolic version of a Picone-type theorem which is based on [4] and an analogue of the Liouville transformations for hyperbolic equations. Motivation for this approach is developed in $\$ 2$ where such transformations are used to establish comparison theorems for SturmLiouville equations. In $\S 3$ we generalize upon the Sturmian theorem of [4], while $\S 4$ exploits a generalized Liouville transformation which, together with the results of $\S 3$, yields Picone-type theorems for a class of nonseparable hyperbolic equations. Examples and 
generalizations are considered in $\S 5$.

2. Picone-type theorems for ODE's via transformations. While the proof of the Sturm-Picone theorem seems to require a tool from the calculus of variations, other forms of this theorem can be obtained from the classical Sturm comparison theorem and the Liouville transformation. In order to compare zeros of solutions of

$$
l[u] \equiv-\left(m(t) u^{\prime}\right)^{\prime}+p(t) u=0
$$

and

$$
L(v) \equiv-\left(M(t) v^{\prime}\right)^{\prime}+P(t) v=0,
$$

when $0<M(t) \leqq m(t)$ and $P(t) \leqq p(t)$ in $[a, b]$, it is natural to try an oscillation-preserving change of variables

$$
u(t)=\rho(t) U(t) ; \quad \tau=\tau(t)
$$

which transforms (2.1) into

$$
-\frac{d}{d \tau}\left(M(t) \frac{d U}{d \tau}\right)+Q(t) U=0
$$

so that both equations have the same principal parts. A direct calculation shows that (2.3) transforms (2.1) into (2.4) whenever

$$
\rho^{2} \frac{d \tau}{d t}=\frac{M}{m}
$$

and that then

$$
Q(t)=\rho l[\rho]\left(\frac{d \tau}{d t}\right)^{-1}
$$

In particular, one can choose $\tau \equiv t$ so that $\rho=M / m$ and $Q=\rho l[\rho]$.

A slight variant of this approach is to require that

$$
0<M(t) \leqq \mu \leqq m(t)
$$

in $[a, b]$ for some constant $\mu$. This approach enables us to transform both (2.1) and (2.2) into equations of the form

$$
\begin{aligned}
& -\mu U_{\tau \tau}+\hat{q}(\tau) U=0 \\
& -\mu V_{\sigma \sigma}+\hat{Q}(\sigma) V=0
\end{aligned}
$$

by means of transformations

$$
\begin{array}{llrl}
u & =\rho U ; & \tau & =\tau(t) \\
v & =R V ; & & \sigma=\sigma(t)
\end{array}
$$


which satisfy

$$
\rho^{2} \frac{d \tau}{d t}=\frac{\mu}{m} ; \quad R^{2} \frac{d \sigma}{d t}=\frac{\mu}{M} .
$$

For example, we could choose $\rho \equiv 1, R \equiv 1$ and

$$
\tau=\gamma+\int_{r}^{t} \frac{\mu}{m(s)} d s ; \quad \sigma=\gamma+\int_{r}^{t} \frac{\mu}{M(s)} d s
$$

for some $\gamma \in\left[t_{1}, t_{2}\right]$. Then $q=m / \mu p, Q=M / \mu P$, and $\tau$ is a contraction while $\sigma$ is a dilation, so that

$$
\left[\tau\left(t_{1}\right), \tau\left(t_{2}\right)\right] \subseteq\left[t_{1}, t_{2}\right] \leqq\left[\sigma\left(t_{1}\right), \sigma\left(t_{2}\right)\right] .
$$

A solution $u(t)$ of (2.1) with $u\left(t_{1}\right)=u\left(t_{2}\right)=0$ now corresponds to a solution $\hat{U}(\tau)$ of (2.5) with zeros at $\tau\left(t_{1}\right)$ and $\tau\left(t_{2}\right)$. If $\hat{Q}(\tau) \leqq \hat{q}(\tau)$ in $\left[\tau\left(t_{1}\right), \tau\left(t_{2}\right)\right]$ then, by Sturm's comparison theorem, $\hat{V}(\sigma)$ has a zero in $\left[\tau\left(t_{1}\right), \tau\left(t_{2}\right)\right]$ while the original solution $v(t)$ of (2.2) must have a zero in $\left[\sigma^{-1}\left(\tau\left(t_{1}\right)\right), \sigma^{-1}\left(\tau\left(t_{2}\right)\right)\right]$. However, in order to assure that $\hat{Q}(\tau) \leqq$ $\hat{q}(\tau)$ one must again assume the existence of a constant $\lambda$ such that

$$
Q(t) \leqq \lambda \leqq q(t)
$$

in $\left[t_{1}, t_{2}\right]$.

This approach, which will cary over to the hyperbolic case, suggests the following definition for functions $f(\boldsymbol{x})$ and $g(\boldsymbol{x})$ defined in a common domain $G \subseteq \boldsymbol{R}^{n}$.

Definition 2.1. We say that $f$ is a strong majorant of $g$ in $G$ if there exists a constant $\lambda$ such that $f(\boldsymbol{x}) \geqq \lambda \geqq g(\boldsymbol{x})$ for all $\boldsymbol{x} \in G$. We then write " $f \gg g$ in $G$."

With this definition one can summarize the preceding discussion with the following Picone-type theorem based on the Liouville transformation.

THEOREM 2.2. Let $u(t)$ and $v(t)$ be nontrivial solutions of (2.1) and (2.2), respectively, satisfying $u\left(t_{1}\right)=u\left(t_{2}\right)=0$. If

$$
0<M \ll m
$$

and

$$
M P \ll m p
$$

in $\left[t_{1}, t_{2}\right]$, then every solution of (2.2) has a zero in

$$
\left[\sigma^{-1}\left(\tau,\left(t_{1}\right)\right), \sigma^{-1}\left(\tau\left(t_{2}\right)\right)\right] \subset\left[t_{1}, t_{2}\right] .
$$


On the one hand the hypotheses involving strong dominance demand more than one would like. They suggest the price to be paid for eschewing mathematical tools rooted in the calculus of variations and, in the present paper, for dealing with the hyperbolic case where such variational tools do not exist.

On the other hand, Theorem 2.2 also has some advantages over the usual Picone theorem. Specifically, there are situations in which we do not have $P(t) \leqq p(t)$ in $\left[t_{1}, t_{2}\right]$ but the fact that $M \ll m$, in $\left[t_{1}, t_{2}\right)$ makes it possible to satisfy $P \ll(m / M) p$ in $\left[t_{1}, t_{2}\right]$. Also whereas the Picone theorem only concludes that $v(t)$ has a zero in $\left(t_{1}, t_{2}\right]$, Theorem 2.2 specifies a proper sub-interval of $\left[t_{1}, t_{2}\right]$ in which $v(t)$ has a zero.

However, our main interest in this approach is that it carries over to a class of nonseparable hyperbolic equations, as will be shown in $\S 4$.

3. A generalized Sturmian theorem. By way of preparation, we consider a pair of hyperbolic equations of the form

$$
\begin{aligned}
& l u \equiv-\left(m(\boldsymbol{x}, t) u_{t}\right)_{t}+\sum_{i, j=1}^{n} D_{i}\left(a_{i j}(\boldsymbol{x}, t) D_{j} u\right)+p(\boldsymbol{x}, t) u=0 \\
& L v \equiv-\left(M(\boldsymbol{x}, t) v_{t}\right)_{t}+\sum_{i, j=1}^{n} D_{i}\left(A_{i j}(\boldsymbol{x}, t) D_{j} v\right)+P(\boldsymbol{x}, t) v=0
\end{aligned}
$$

in a smooth bounded domain $G \leqq \boldsymbol{R}^{n+1}$. We assume that $m$ and $M$ are of class $C^{1}$ and positive, that the matrices $a=\left(a_{i j}\right)$ and $A=\left(A_{i j}\right)$ are $C^{1}$, symmetric and positive definite, and that $p$ and $P$ are continuous in $G$.

In order to apply Green's theorem to (3.1) it is convenient to define

$$
\begin{aligned}
\nabla u & =\left(D_{1} u, \cdots, D_{n} u, u_{t}\right), \\
\boldsymbol{n} & =\left(\sum_{i=1}^{n} a_{i 1} \nu_{i}, \cdots, \sum_{i=1}^{n} a_{i n} \nu_{i},-m \nu_{n+1}\right)
\end{aligned}
$$

where $\nu=\left(\nu_{1}, \cdots, \nu_{n+1}\right)$ is the exterior unit normal to $\partial G$. Then we have

$$
\iint_{G} v l u d \boldsymbol{x} d t=\int_{\partial G} v \nabla u \cdot n d s-\iint_{G}\left[\nabla u \cdot\left(\begin{array}{rr}
a & -m \\
0 & 0
\end{array}\right) \nabla v-p u v\right] d \boldsymbol{x} d t
$$

The boundary integral in (3.3( motivates a decomposition of $\partial G$ into

$$
\gamma_{1}=\{(\boldsymbol{x}, t) \in \partial G \mid \nu \cdot n \leqq 0 \text { at }(\boldsymbol{x}, t)\}
$$




$$
\gamma_{2}=\{(\boldsymbol{x}, t) \in \partial G \mid \nu \cdot \boldsymbol{n}>0 \text { at }(\boldsymbol{x}, t)\} .
$$

As shown in [4], this decomposition corresponds to the "space-like" and "time-like" components of $\partial G$ relative to (3.1). More geomertically, under a canonical change of coordinates which yields $a_{i j}=\delta_{i j}$ and $m=1$ at a given $(\boldsymbol{x}, t)=\left(\boldsymbol{x}_{0}, t_{0}\right)$ we consider a particular tangent vector

$$
\begin{aligned}
\boldsymbol{T}\left(\boldsymbol{x}_{0}, t_{0}\right) & =\left(\nu_{1}, \cdots, \nu_{n},-\frac{\nu_{1}^{2}+\cdots+\nu_{n}^{2}}{\nu_{n+1}}\right) & & \left(\nu_{n+1} \neq 0\right) \\
& =\left(\nu_{1}, \cdots, \nu_{n}, 0\right) & & \left(\nu_{n+1}=0\right) .
\end{aligned}
$$

If $\mathscr{C}\left(\boldsymbol{x}_{0}, t_{0}\right)$ denotes the interior of the characteristic cone emanating from $\left(\boldsymbol{x}_{0}, t_{0}\right)$, then $\boldsymbol{T} \in \mathscr{C}$ if and only if $\left(\boldsymbol{x}_{0}, t_{0}\right) \in \gamma_{2}$.

An analogous development relative to (3.2), with

$$
N=\left(\sum_{i=1}^{n} \mathrm{~A}_{i 1} \nu_{i}, \cdots, \sum_{i=1}^{n} A_{i n} \nu_{i},-M \nu_{n+1}\right)
$$

yields

$$
\iint_{G} u L v d \boldsymbol{x} d t=\int_{\partial G} u \nabla v \cdot N d s-\iint_{G}\left[\nabla v \cdot\left(\begin{array}{rr}
\mathrm{A} & 0 \\
0 & -m
\end{array}\right) \nabla u-P u v\right] d \boldsymbol{x} d t .
$$

This equation motivates a second decomposition of $\partial G$ into

$$
\begin{aligned}
& \Gamma_{1}=\{(\boldsymbol{x}, t) \in \partial G \mid \nu \cdot N \leqq 0 \text { at }(x, t)\} \\
& \Gamma_{2}=\{(\boldsymbol{x}, t) \in \partial G \mid \nu \cdot N>0 \text { at }(x, t)\}
\end{aligned}
$$

corresponding to the "space-like" and "time-like" components of $\partial G$ relative to (3.2).

An immediate consequence of these definitions is the following

Lemma 3.1. If for all $(\boldsymbol{x}, t) \in \bar{G}$

$$
0<M(\boldsymbol{x}, t) \leqq m(\boldsymbol{x}, t)
$$

and

$$
\begin{aligned}
& 0<\sum a_{i j}(\boldsymbol{x}, t) \eta_{i} \eta_{j} \leqq \sum A_{i j}(\boldsymbol{x}, t) \eta_{i} \eta_{j} \\
& \quad \text { for all real } n \text {-tuples }\left(\eta_{1}, \cdots, \eta_{n}\right) \neq(0, \cdots, 0) \text {, then }
\end{aligned}
$$

$\Gamma_{1} \cong \gamma_{1}$ and $\Gamma_{2} \supseteqq \gamma_{2}$.

Proof. By definition, $(\boldsymbol{x}, t) \in \gamma_{2}$ if and only if $\sum a_{i j}(\boldsymbol{x}, t) \nu_{i} \nu_{j}>$ $m(\boldsymbol{x}, t) \nu_{n+1}^{2}$ which clearly implies that $\sum A_{i j}(\boldsymbol{x}, t) \nu_{i} \nu_{j}>M(\boldsymbol{x}, t) \nu_{n+1}^{2}$ and that $(\boldsymbol{x}, t) \in \Gamma_{2}$. A similar argument shows that $\Gamma_{1} \subseteq \gamma_{1}$.

Corollary 3.2. $\partial G \subset \gamma_{1} \cup \Gamma_{2}$. 
With this background, we consider the more special case of functions $U(\boldsymbol{x}, t), V(\boldsymbol{x}, t)$ which are, respectively, solutions in $G \subset \boldsymbol{R}^{n+1}$ of

$$
\begin{aligned}
& -\left(m(\boldsymbol{x}, t) U_{t}\right)_{t}+\sum_{i, j=1}^{n} D_{i}\left(a_{i j}(\boldsymbol{x}, t) D_{j} U\right)+q(\boldsymbol{x}, t) U=0 \\
& -\left(m(\boldsymbol{x}, t) V_{t}\right)_{t}+\sum_{i, j=1}^{n} D_{i}\left(a_{i j}(\boldsymbol{x}, t) D_{j} V\right)+Q(\boldsymbol{x}, t) V=0,
\end{aligned}
$$

and let $\gamma_{1}^{\prime} \cup \gamma_{2}^{\prime}$ denote the decomposition of $\partial G$ relative to $(3.1)^{\prime}$ or $(3.2)^{\prime}$. In order to show that the inequality

$$
Q(\boldsymbol{x}, t) \leqq q(\boldsymbol{x}, t) \quad \text { for all } \quad(\boldsymbol{x}, t) \in G
$$

assures that $V(\boldsymbol{x}, t)$ changes sign in a domain $G$ in which $U(\boldsymbol{x}, t) \neq 0$, we seek to show the incompatibility of the conditions

(i) $Q(x, t) \leqq q(x, t)$ for all $(x, t) \in G,(P \not \equiv Q)$

(ii) $U(x, t)>0$ in $G$,

(iii) $V(x, t)>0$ in $G$.

The desired contradiction follows from the fact that for solutions $U$ and $V$ of $(3.1)^{\prime}$ and (3.2)', respectively, Green's theorem yields

$$
\iint_{G}(q-Q) U V d \boldsymbol{x} d t=-\int_{\partial G}(V \nabla U \cdot \boldsymbol{n}-U \nabla V \cdot \boldsymbol{n}) d \boldsymbol{s} .
$$

In the event that (i)-(iii) hold and

$$
V=0 \text { on } \gamma_{2}^{\prime} \text { and } U=0 \text { on } \gamma_{1}^{\prime}
$$

then (3.8) cannot hold and we have the following result.

THEOREM 3.3. Let $U(\boldsymbol{x}, t)$ and $V(\boldsymbol{x}, t)$ be solutions of $(3.1)^{\prime}$ and $(3.2)^{\prime}$, respectively, satisfying $U>0$ in $G, U=0$ on $\gamma_{1}^{\prime}$ and $V=0$ on $\gamma_{2}^{\prime}$. If (3.7) is satisfied in $G$ with $Q \not \equiv q$, then $V(x, t)$ changes sign in $G$.

4. A Picone-type theorem. We turn now to the more difficult task of comparing two equations of the form (3.1) and (3.2) with different principal parts. By way of physical motivation, we note that (3.1) can be interpreted as describing the oscillations (about $u=0$ ) of a vibrating system of density $m(x, t)$, whose elastic properties are given by the matrix $\left(a_{i j}\right)$, and which is subject to a linear restoring force $-p(\boldsymbol{x}, t) u$. Intuitively, we would expect that a decrease in density and an increase in the elastic and restoring forces should lead to more rapid oscillation about $u=0$. This is what motivates us to consider special cases of (3.1) and (3.2) subject to the hypotheses (3.5), (3.6), and (3.7), seeking conditions under which a solution $v(x, t)$ of (3.2) must change sign in a particular 
domain $G$.

Our principal result deals with a pair of nonseparable hyperbolic equations

$$
\begin{aligned}
& l u \equiv-\left(m(t) u_{t}\right)_{t}+\left(a(x) u_{x}\right)_{x}+p(x, t) u=0 \\
& L v \equiv-\left(M(t) v_{t}\right)_{t}+\left(A(x) v_{x}\right)_{x}+P(x, t) v=0
\end{aligned}
$$

for which there exist constants $\mu$ and $\alpha$ such that

$$
\begin{aligned}
& 0<M(t) \leqq \mu \leqq m(t) \\
& 0<a(x) \leqq \alpha \leqq A(t)
\end{aligned}
$$

in a smooth bounded domain $G \subset \boldsymbol{R}^{n+1}$. In order to establish boundary conditions which will assure that $v(x, t)$ changes sign in $G$ we seek a generalized Liouville transformation which will enable us to apply Theorem 3.3 to the transformed equations. Motivated by a technique described by Ahlbrandit, Hinton, and Lewis in [1], we consider the functional

$$
J[u]=\iint_{G}\left(m u_{t}^{2}-a u_{x}^{2}+p u^{2}\right) d x d t
$$

associated with the differential operator $l$ of (4.1). It is readily verified that the substitution $u(x, t)=\rho(x, t) U(x, t)$ yields

$$
\begin{aligned}
J[\rho U] & =\iint_{G}\left(m \rho^{2} U_{t}^{2}-a \rho^{2} U_{x}^{2}+\rho l[\rho] U^{2}\right) d x d t \\
& =\oint_{\partial G}\left(\frac{m}{2} \rho^{2}\right)_{t} U^{2} d x+\left(\frac{a}{2} \rho^{2}\right)_{x} U^{2} d t .
\end{aligned}
$$

Neglecting the boundary term, but making a change of variables $\xi=\xi(x), \tau=\tau(t)$ yields

$$
J[\rho U]=\iint_{\tilde{G}}\left[m \rho^{2} \frac{\tau_{t}}{\xi_{x}} U_{=}^{2}-a \rho^{2} \frac{\xi_{x}}{\tau_{t}} U_{\tilde{\xi}}^{2}+\frac{1}{\xi_{x} \tau_{t}} \rho l[\rho] U^{2}\right] d \xi d \tau
$$

where we have made use of the fact that $J(x, t / \xi, \tau)=1 / \xi_{x} \tau_{t}$. Anticipating the form of the Euler-Lagrange equation associated with $J[\rho U]$, we impose the conditions

$$
m \rho^{2} \frac{\tau_{t}}{\xi_{x}}=\mu ; \quad a \rho^{2} \frac{\xi_{x}}{\tau_{t}}=\alpha
$$

which are satisfied if

$$
\rho=\sqrt[4]{\frac{\alpha \mu}{a m}} ; \quad \xi_{x}=\sqrt{\frac{\alpha}{a}} ; \quad \tau_{t}=\sqrt{\frac{\mu}{m}} .
$$


Then, as can be verified by a direct calculation, (4.1) is transformed into

$$
\tilde{l}[U] \equiv-\mu U_{\tau \tau}+\alpha U_{\xi \xi}+\left(\frac{l[\hat{\rho}]}{\rho}\right) U=0 .
$$

Analogously, the transformation

$$
v=\kappa V ; \quad \eta=\eta(x) ; \quad \sigma=\sigma(t)
$$

with

$$
\kappa=\sqrt{\frac{\alpha \mu}{\mathrm{AM}}} ; \quad \eta_{x}=\sqrt{\frac{\alpha}{A}} ; \quad \sigma_{t}=\sqrt{\frac{\mu}{M}}
$$

transforms (4.2) into

$$
L[V]=-\mu V_{\sigma \sigma}+\alpha V_{\eta \eta}+\left(\frac{L[\hat{\kappa}]}{\kappa}\right) V=0 .
$$

With this machinery we are able to establish our principal result.

TheOrem 4.1. Suppose $G$ is a smooth bounded domain which is convex in $\boldsymbol{R}^{2}$ and that $u(x, t)$ is a solution of (4.1) satisfying $u=0$ on $\gamma_{1}$. If there exist constants $\mu$ and $\alpha$ such that

$$
\begin{aligned}
& 0<M(t) \leqq \mu \leqq m(t) \\
& 0<a(x) \leqq \alpha \leqq A(x) \\
& \frac{L[\kappa]}{[\kappa]} \ll \frac{l[\rho]}{\rho} \quad(\text { not identically equal) }
\end{aligned}
$$

for all $(x, t)$ in $G$, then every solution $v(x, t)$ of (4.2) satisfying $v=0$ on $\Gamma_{2}$ changes sign in $G$.

Proof. Choose a point $(\varepsilon, \delta) \in G$ so that

$$
\xi=\varepsilon+\int_{\varepsilon}^{\infty} \sqrt{\frac{\alpha}{a(s)}} d s ; \quad \tau=\delta+\int_{\delta}^{t} \sqrt{\frac{\mu}{m(s)}} d s
$$

satisfy (4.6). From the hypotheses it follows that (4.10) is a dilation in $x$ and a contraction in $t$ which leaves $(\varepsilon, \delta)$ fixed. Analogously,

$$
\eta=\varepsilon+\int_{\varepsilon}^{x} \sqrt{\frac{\alpha}{A(s)}} d s ; \quad \sigma=\delta+\int_{\delta}^{t} \sqrt{\frac{\mu}{M(s)}} d s
$$

is a contraction in $x$ and a dilation in $t$ which satisfies (4.8) and also leaves $(\varepsilon, \delta)$ fixed. Denoting the images of $G$ under (4.10) and (4.11) by $\widetilde{H}$ and $\tilde{H}$, respectively, it follows from the convexity of $G$ that 
$\tilde{H} \cap \tilde{H} \subset G$. Denoting this intersection by $\widetilde{G}$, we consider the decomposition of $\partial \widetilde{G}$ into its "space-like" and "time-like" components

$$
\partial \widetilde{G}=\widetilde{\Gamma}_{1} \cup \widetilde{\Gamma}_{2} .
$$

Since the property of being "space-like" is invariant under a change of variables, it follows from Corollary 3.2 that $\widetilde{\Gamma}_{1}$ is contained in the image of $\gamma_{1}$ under (4.10) and that $U(\xi, \tau)=0$ on $\widetilde{\Gamma}_{1}$. By an analogous argument we have $V(\eta, \sigma)=0$ on $\widetilde{\Gamma}_{2}$. Since $U$ and $V$ satisfy (4.7) and (4.9) respectively, an application of Theorem 3.3 (in which we identify $\xi$ and $\eta$ with $x, \tau$ and $\sigma$ with $t$ ) yields the conclusion that $V$ changes sign in $\widetilde{G}$. Therefore $v$ changes sign in the pre-imge of $\widetilde{G}$ under (4.11), and this completes the proof.

5. Remarks and examples. Unless (4.10) and (4.11) are identity mappings, Theorem 4.1 yields a specific proper subset of $G$ in which a zero of $v$ must occur; this is stronger than the usual Sturmian conclusion. The assumption that $G$ be convex can be weakened to the requirement that it be closed under horizontal and vertical contractions.

A natural choice for (4.1) is the wave equation

$$
l u \equiv-u_{t t}+u_{x x}+p(t) u=0 .
$$

If (5.1) is subject to boundary conditions of the form $u\left(x_{1}, t\right)=$ $u\left(x_{2}, t\right)=0$, then it allows a separation of variables $u=X(x) T(t)$ so that $u(x, t)$ has a rectangular nodal domain $G=\left\{(x, t) \mid x_{1}<x<x_{2}\right.$, $\left.t_{1}<t<t_{2}\right\}$ whenever $T^{\prime \prime}+\left(\pi^{2} /\left(x_{2}-x_{1}\right)^{2}-p(t)\right) T=0$ is not disconjugate on $\left(t_{1}, \infty\right)$. The techniques of [2], [3], [10], and [12] show that every solution of

$$
\begin{gathered}
-v_{t t}+v_{x x}+P(x, t) v=0 \\
v\left(x_{1}, t\right)=v\left(x_{2}, t\right)=0
\end{gathered}
$$

changes sign in $G$ whenever $P(x, t) \leqq p(t)(P \not \equiv p)$ in $G$. However Theorem 4.1 enables one to establish zeros for solutions of certain equations of the form

$$
\begin{gathered}
-\left(M(t) v_{t}\right)_{t}+\left(A(x) v_{x}\right)_{x}+P(x, t) v=0 \\
v\left(x_{1}, t\right)=v\left(x_{2}, t\right)=0
\end{gathered}
$$

when $M(t) \leqq 1$ in $\left(t_{1}, t_{2}\right)$ and $A(x) \geqq 1$ in $\left(x_{1}, x_{2}\right)$. In this situation we can choose $\alpha=\mu=1$ so that $\xi=x, \tau=t, \rho \equiv 1, \kappa=\sqrt[4]{1 / A M}$, $\eta_{x}=\sqrt{1 / A}$, and $\rho_{t}=\sqrt{1 / M}$.

In case $p(t)$ is a negative constant (i.e., $p(t) \equiv-p_{0}$ ), equation (5.1) also has the solution $u=J_{0}\left(\sqrt{p_{0}}\left(t^{2}-x^{2}\right)\right)$. Now $u(x, t)$ has 
hyperbolic nodal lines of the form

$$
t^{2}-x^{2}=\frac{j_{n}}{\sqrt{p_{0}}}
$$

where $\left\{j_{n}\right\}$ are the zeros of the Bessel function of order zero. This solution does not allow a separation of variables in $x$ and $t$ nor does it have any bounded nodal domains. Yet Theorem 4.1 applies to solutions $v(x, t)$ of (5.2) in a domain $G$ bounded by two hyperbolic nodal lines and a pair of space-like curves (such as $x t=$ const) provided $v(x, t)=0$ on the bounding space-like curves.

Finally, we note that when $p(t) \equiv 0,(5.1)$ has the solutions

$$
u=\left(x-x_{0}\right)^{2}+\left(t-t_{0}\right)^{2}-r^{2}
$$

which generate circular nodal domains with arbitrary centers and radii. With such solutions we can apply Theorem 4.1 to solutions of (5.2) which vanish on the space-like boundary of an arbitrary circle in the $(x, t)$-plane whenever $M(t) \leqq 1, A(x) \geqq 1$ and $L[\kappa] / \kappa \ll$ $l[\rho] / \rho$.

The extension of Theorem 4.1 to a higher number of independent variables is straightforward as long as the principal part is appropriately restricted-e.g.

$$
l[u] \equiv-\left(m(t) u_{t}\right)_{t}+\left(a(x) u_{x}\right)_{x}+\left(b(y) u_{y}\right)_{y}+p(x, y, t) u=0 .
$$

Of greater interest is the question of whether one can handle the two-dimensional case where $m=m(x, t)$ and/or $a=a(x, t)$. The techniques pursued above suggest that such results might follow from a study of generalized Liouville transformations corresponding to more general principal parts than those considered here.

\section{REFERENCES}

1. C. D. Ahlbrandt, D. B. Hinton, and Roger T. Lewis, The effect of a variable change on oscillation and disconjugacy criteria, with applications to spectral theory and asymptotic theory, Proceedings of the 1980 Dundee conference on differential equations, to appear.

2. C. Kahane, Oscillation theorems for solutions of hyperbolic equations, Proc. Amer. Math. Soc., 41 1973), 183-188.

3. K. Kreith, Sturmian theorems for hyperbolic equations, Proc. Amer. Math. Soc., 22 (1969), 277-281.

4. - A Sturm theorem for partial differential equations of mixed type, Proc. Amer. Math. Soc., 81 (1981), 75-78.

5. W. Leighton, A substitute for the Picone formula, Bull. Amer. Math. Soc., 55 (1949), $325-328$.

6. M. Picone, Sui valori eccezionali di un parametro da cui dipende un aquazione differenziale lineare ordinaria dəl second'ordine, Ann. Scuola Norm. Pisa, 11 (1909), 1-141. 
7. M. Picone, Un teorema sulle soluzioni delle equazioni lineari ellitiche autoaggiunte alle derivate parziali del secondo-ordine, Atti Accad. Naz. Lincei, 20 (1911), 213-219.

8. W. T. Reid, Ordinary Differential Equations, Wiley, New York, 1971.

9. C, Sturm, Sur les equations differentialles lineaires du second ordre, J. Math. Pures Appl., 1 (1836), 100-186.

10. C. A. Swanson, A dichotomy of Sturmian theory, SIAM Review, 20 (1978), 285-300. 11. Comparison and Oscillation Theory of Linear Differential Equations, Academic Press, New York, 1968.

12. C. C. Travis, Comparison and oscillation theorems for hyperbolic equations, Utilitas Math., 6 (1974), 139-151.

Received January 13, 1981 and in revised form May 18, 1981. Research supported by a grant of the National Science Foundations, MCS 9002130.

UNIVERSITY OF CALIFORNIA

DAVIS, CA 95616 



\section{PACIFIC JOURNAL OF MATHEMATICS}

\section{EDITORS}

DONALD BABBITT (Managing Editor)

University of California

Los Angeles, CA 90024

Hugo RossI

University of Utah

Salt Lake City, UT 84112

C. C. MOORE and ARThur Agus

University of California

Berkeley, CA 94720
J. DugundJI

Department of Mathematics

University of Southern California

Los Angeles, CA 90007

R. FINN and J. MILGRAM

Stanford University

Stanford, CA 94305

\section{ASSOCIATE EDITORS}
R. ARENS
E. F. BeCKENBACH
B. H. NeumanN
F. WOLF
K. YoSHIDA

\section{SUPPORTING INSTITUTIONS}

UNIVERSITY OF ARIZONA

UNIVERSITY OF BRITISH COLUMBIA

CALIFORNIA INSTITUTE OF TECHNOLOGY

UNIVERSITY OF CALIFORNIA

MONTANA STATE UNIVERSITY

UNIVERSITY OF NEVADA, RENO

NEW MEXICO STATE UNIVERSITY

OREGON STATE UNIVERSITY

\author{
UNIVERSITY OF OREGON \\ UNIVERSITY OF SOUTHERN CALIFORNIA \\ STANFORD UNIVERSITY \\ UNIVERSITY OF AAWAII \\ UNIVERSITY OF TOKYO \\ UNIVERSITY OF UTAH \\ WASHINGTON STATE UNIVERSITY \\ UNIVERSITY OF WASHINGTON
}

The Supporting Institutions listed above contribute to the cost of publication of this Journal, but they are not owners or publishers and have no responsibility for its content or policies,

Mathematical parers intended for publication in the Pacific Journal of Mathematics should be in typed form or offset-reproduced, (not dittoed), double spaced with large margins. Please do not use built up fractions in the text of the manuscript. However, you may use them in the displayed equations. Underline Greek letters in red, German in green, and script in blue. The first paragraph or two must be capable of being used separately as a synopsis of the entire paper. Please propose a heading for the odd unmbered pages of less than 35 characters. Manuscripts, in triplicate, may be sent to any one of the editors. Please classify according to the scheme of Math. Reviews, Index to Vol. 39. Supply name and address of author to whom proofs should be sent. All other communications should be addressed to the managing editor, or Elaine Barth, University of California, Los Angeles, California, 90024.

50 reprints to each author are provided free for each article, only if page charges have been substantially paid. Additional copies may be obtained at cost in multiples of 50 .

The Pacific Journal of Mathematics is issued monthly as of January 1966, Regular subscription rate: $\$ 114.00$ a year (6 Vol., 12 issues). Special rate: $\$ 57.00$ a year to individual members of supporting institution.

Subscriptions, orders for numbers issued in the last three calendar years, and changes of address shoud be sent to Pacific Journal of Mathematics, P.O. Box 969, Carmel Valley, CA 93924, U.S.A. Old back numbers obtainable from Kraus Periodicals Co., Route 100, Millwood, NY 10546.

\section{PUBLISHED BY PACIFIC JOURNAL OF MATHEMATICS, A NON-PROFIT CORPORATION}

Printed at Kokusai Bunken Insatsusha (International Academic Printing Co., Ltd.). 8-8, 3-chome, Takadanobaba, Shinjuku-ku, Tokyo 160, Japan. 


\section{Pacific Journal of Mathematics}

\section{Vol. 102, No. 2 \\ February, 1982}

Richard A. Boyce, Irreducible representations of finite groups of Lie type through block theory and special conjugacy classes ...............253

Robert Jay Daverman and Dennis J. Garity, Intrinsically

$(n-2)$-dimensional cellular decompositions of $E^{n} \ldots \ldots \ldots \ldots 275$

Juan Ferrera, Spaces of weakly continuous functions ................285

William George Frederick, $\mu$-theta functions ................... 293

Christopher George Gibson and T. D. Ward, On stratifying pairs of linear

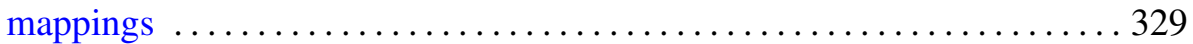

Stanley Joseph Gurak, Minimal polynomials for Gauss circulants and cyclotomic units ........................................ 347

Joachim Georg Hartung, On two-stage minimax problems ............. 355

Robert P. Kaufman, Hausdorff measure, BMO, and analytic functions . . . . 369

Neal I. Koblitz, $p$-adic analog of Heine's hypergeometric $q$-series . . . . . . . 373

Kurt Kreith, Picone-type theorems for hyperbolic partial differential

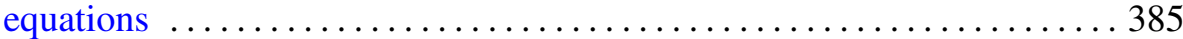

Nicholas J. Kuhn, The geometry of the James-Hopf maps ............. 397

Donald Michael Redmond, Explicit formulae for a class of Dirichlet

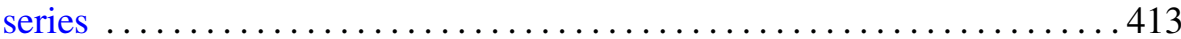

J. R. Respess and Elliott Ward Cheney, Jr., Best approximation problems in tensor-product spaces . .............................. 437

Allen Ross Schweinsberg, The operator equation $A X-X B=C$ with normal $A$ and $B$

Hans-Willi Siegberg and Guentcho Svetoslavov Skordev, Fixed point index and chain approximations

Kondagunta Sundaresan, Geometry and nonlinear analysis in Banach spaces 